ACR70 responses, health outcome measures, CRP levels, and overall incidence of adverse events among all groups. Fortyfour subjects $(15.1 \%)$ discontinued the study, and the rate of discontinuation was similar among treatment groups. Though well-tolerated and safe, ERB-041 failed to demonstrate antiinflammatory efficacy in RA patients, despite evidence of strong activity in preclinical arthritis models. Taken together, these 2 studies suggest that selective ER agonism (both ER $\alpha$ and $\operatorname{ER} \beta$ ) would not have effects on regulation of inflammatory response in RA.

There are several possible explanations. ERs are nuclear hormone receptors that can either directly bind to estrogen response elements in gene promoters or serve as cofactors with other transcription factors (i.e., NF- $\kappa \mathrm{B}$ /activator protein 1) (5). Cytoplasmic ER and membrane-associated ER affect specific kinase-signaling pathways. ERs have prominent effects on immune function in both the innate and adaptive immune responses. However, the roles of estrogen receptors may be different between species, significantly limiting the easy translation of preclinical studies (in animal models) into the clinical setting (6).

Second, the role of estrogens in RA is the subject of debate since both proinflammatory and antiinflammatory effects have been reported; important evidence of their dual role is provided by their peripheral conversion to various proinflammatory or antiinflammatory metabolites at the level of the RA synovial tissue. Recently, using estrone and $17 \beta$-estradiol as substrates, the production of $16 \alpha-, 4-$, and 2-hydroxylated estrogens and their 4- and 2-methylation products in RA and osteoarthritis (OA) synovial cells was evaluated (7). The levels of $16 \alpha$-hydroxylated estrone/17 $\beta$-estradiol $(16 \alpha \mathrm{OH}$-estrone/ $16 \alpha \mathrm{OH}-17 \beta$-estradiol) were higher than the levels of all other estrogen metabolites. RA synovial cells produced more $16 \alpha \mathrm{OH}$-estrone than did OA synovial cells. Importantly, the $16 \alpha \mathrm{OH}$-estrones did not inhibit tumor necrosis factor (TNF) secretion, whereas all other estrogen metabolites had marked inhibitory effects. These and similar findings indicate that precursor estrogens are converted into proinflammatory metabolites, particularly in RA synovial cells. RA synovial cells mainly produce the proproliferative $16 \alpha \mathrm{OH}$-estrone, which, in addition to $16 \alpha \mathrm{OH}-17 \beta$-estradiol, is one of the only two estrogens studied that does not inhibit TNF secretion (8). A preponderance of $16 \alpha$-hydroxyestrone is an unfavorable sign in RA synovial inflammation.

Therefore, it must be considered that, even in the presence of a normal interaction between endogenous estrogens (or agonists) and their functional ERs, an altered balance in peripheral estrogen metabolites might induce different cell (and clinical) responses in RA patients and promote unexpected effects.

Maurizio Cutolo, MD University of Genoa Genoa, Italy

1. Van Vollenhoven RF, Houbiers JG, Buttgereit F, Hout J, Boers $\mathrm{M}$, Leij S, et al. The selective estrogen receptor $\alpha$ agonist Org 37663 induces estrogenic effects but lacks antirheumatic activity: a phase IIa trial investigating efficacy and safety of Org 37663 in postmenopausal female rheumatoid arthritis patients receiving stable background methotrexate or sulfasalazine. Arthritis Rheum 2010;62:351-8.
2. Prevoo ML, van 't Hof MA, Kuper HH, van Leeuwen MA, van de Putte LB, van Riel PL. Modified disease activity scores that include twenty-eight-joint counts: development and validation in a prospective longitudinal study of patients with rheumatoid arthritis. Arthritis Rheum 1995;38:44-8.

3. Roman-Blas JA, Castaneda S, Cutolo M, Herrero-Beaumont G. Efficacy and safety of a selective estrogen receptor-beta agonist, ERB-041, in patients with rheumatoid arthritis. Arthritis Care Res (Hoboken) 2010;62:1588-93.

4. Felson DT, Anderson JJ, Boers M, Bombardier C, Furst D, Goldsmith C, et al. American College of Rheumatology preliminary definition of improvement in rheumatoid arthritis. Arthritis Rheum 1995;38:727-35.

5. Cunningham M, Gilkeson G. Estrogen receptors in immunity and autoimmunity. Clin Rev Allergy Immunol 2010. E-pub ahead of print.

6. Cutolo M. Sex and rheumatoid arthritis: mouse model versus human disease [editorial]. Arthritis Rheum 2007;56:1-3.

7. Schmidt M, Hartung R, Capellino S, Cutolo M, Pfeifer-Leeg A, Straub RH. Estrone $/ 17 \beta$-estradiol conversion to, and tumor necrosis factor inhibition by, estrogen metabolites in synovial cells of patients with rheumatoid arthritis and patients with osteoarthritis. Arthritis Rheum 2009;60:2913-22.

8. Cutolo M, Straub RH, Bijlsma JW. Neuroendocrine-immune interactions in synovitis. Nat Clin Pract Rheumatol 2007;3:627-34.

DOI 10.1002/art.27724

\section{The heart in rheumatoid arthritis: contrasting or misleading data from clinical research? Comment on the article by Giles et al}

\section{To the Editor:}

We read with great interest the article by Giles et al (1), who recently reported that the progression to heart failure in patients with rheumatoid arthritis (RA) may occur through reduced myocardial mass rather than through hypertrophy. Cardiac magnetic resonance imaging (MRI) was utilized as the method for measuring left ventricular structure and function. However, we believe that some methodologic problems undermine the strength of this research and its results.

First, the RA cohort in the Evaluation of Subclinical Cardiovascular Disease and Predictors of Events in Rheumatoid Arthritis (ESCAPE RA) study was enrolled from October 2004 through May 2006, and the patients were recruited from the Johns Hopkins Arthritis Center, where they were receiving treatment. Giles and colleagues state that only their 75 study subjects from the selected subset of the ESCAPE RA patients (predefined as $40 \%$ of the RA patient sample) were scanned with cardiac MRI. In contrast, the non-RA control subjects were recruited between 2000 and 2002, from 1,086 subjects of the Baltimore Multi-Ethnic Study of Atherosclerosis, a specific subset (out of a total 6,814 subjects from 6 US communities) that had been enrolled at the Johns Hopkins Field Center. Only 788 of these control patients underwent examination by cardiac MRI, and of those 788, only 225 were included in Giles and colleagues' report (1). The matching of RA patients with 3 control subjects each for comparison was randomized (though Giles and colleagues did not describe their randomization method in detail). The fact that the 2 groups were 
enrolled at 2 different times and from 2 different studies may have resulted in selection bias, thereby invalidating the results of the study (2).

Moreover, some confounding factors (e.g., duration of both hypertension and diabetes in the 2 groups) were not considered. Cardiac MRI was recommended by Giles et al as an imaging method that avoids overestimation of the left ventricular mass in the setting of eccentric left ventricular hypertrophy, a concern that is often relevant in RA. However, there are few reports on the comparison of cardiac MRI with transthoracic echocardiography, and a recent study has shown that they have similar reliability in monitoring left ventricular mass (3). Thus, echocardiography can be considered a wellrecognized technique for the morphologic and functional evaluation of the left ventricle for both clinical and research purposes, including evaluations of RA patients.

Rudominer et al recently published a report elaborating on this and describing the association of RA with increased left ventricular mass (4). Their findings are consistent with those of our previous studies of RA patients $(5,6)$. Patients with RA have both structural and functional left ventricular involvement. In RA patients, we have previously demonstrated that diastolic dysfunction (detected as relaxation abnormalities during left ventricular filling) is directly correlated with structural changes in the left ventricle, specifically, changes involving left ventricular mass, interventricular septal thickness, and left ventricular posterior wall thickness (6). All of these seem to be features of the systemic disease process in RA, and diastolic dysfunction seems to be a consequence of left ventricular structural changes. An autopsy-based study revealed structural cardiac involvement (7). In a meta-analysis of previously published reports (8) we ascertained the effect size of RA on left ventricular mass index in patients compared with healthy controls; comparison was rigorous in the studies considered in the meta-analysis. Moreover, other studies contribute to the growing body of research on this topic, confirming structural left ventricular involvement in RA $(9,10)$. In conclusion, we believe that the data collected by Giles and colleagues mislead the scientific community, while a large amount of research confirms that left ventricular structural and functional changes are one of the established clinical features of RA.

\section{Salvatore Corrao, MD \\ Luigi Calvo, MD \\ University of Palermo \\ Christiano Argano, MD \\ Fondazione San Raffaele-G.Giglio di Cefalù \\ Giuseppe Licata, MD \\ University of Palermo \\ Palermo, Italy}

1. Giles JT, Malayeri AA, Fernandes V, Post W, Blumenthal RS, Bluemke D, et al. Left ventricular structure and function in patients with rheumatoid arthritis, as assessed by cardiac magnetic resonance imaging. Arthritis Rheum 2010;62:940-51.

2. Kleinbaum DG, Morgenstern H, Kupper LL. Selection bias in epidemiologic studies. Am J Epidemiol 1981;113:452-63.

3. Guenzinger R, Wildhirt SM, Voegele K, Wagner I, Schwaiger M, Bauernschmitt R, et al. Comparison of magnetic resonance imaging and transthoracic echocardiography for the identification of LV mass and volume regression indices 6 months after mitral valve repair. J Card Surg 2008;23:126-32.

4. Rudominer RL, Roman MJ, Devereux RB, Paget SA, Schwartz
JE, Lockshin MD, et al. Independent association of rheumatoid arthritis with increased left ventricular mass but not with reduced ejection fraction. Arthritis Rheum 2009;60:22-9.

5. Corrao S, Salli L, Arnone S, Scaglione R, Amato V, Cecala M, et al. Cardiac involvement in rheumatoid arthritis: evidence of silent heart disease. Eur Heart J 1995;16:253-6.

6. Corrao S, Salli L, Arnone S, Scaglione R, Pinto A, Licata G. Echo-Doppler left ventricular filling abnormalities in patients with rheumatoid arthritis without clinically evident cardiovascular disease. Eur J Clin Invest 1996;26:293-7.

7. Koivuniemi R, Paimela L, Suomalainen R, Tornroth T, LeirisaloRepo M. Amyloidosis is frequently undetected in patients with rheumatoid arthritis. Amyloid 2008;15:262-8.

8. Corrao S, Scaglione R, Calvo L, Licata G. A meta-analysis of the effect size of rheumatoid arthritis on left ventricular mass [letter]. Arthritis Rheum 2009;60:2851-2.

9. Arslan S, Bozkurt E, Sari RA, Erol MK. Diastolic function abnormalities in active rheumatoid arthritis evaluation by conventional Doppler and tissue Doppler: relation with duration of disease. Clin Rheumatol 2006;25:294-9.

10. Gonzalez-Juanatey C, Testa A, Garcia-Castelo A, Garcia-Porrua C, Llorca J, Ollier WE, et al. Echocardiographic and Doppler findings in long-term treated rheumatoid arthritis patients without clinically evident cardiovascular disease. Semin Arthritis Rheum 2004;33:231-8.

DOI 10.1002/art.27723

\section{Reply}

\section{To the Editor:}

We thank Dr. Corrao and colleagues for their letter. They question whether issues of bias may have impugned our finding that left ventricular mass, assessed using cardiac MRI, was on average $18 \%$ lower among RA patients, compared with a demographically matched control group, both without known cardiovascular disease (CVD). Furthermore, they assert the validity of echocardiography for the comparison of left ventricular mass between RA and non-RA groups.

To clarify, ESCAPE RA is an ancillary study of the Baltimore Multi-Ethnic Study of Atherosclerosis (MESA) and not a different study. ESCAPE RA participants undergo the same testing, using the same protocols, facilities, equipment, and quality control measures as MESA. In addition, data are managed by the same coordinating center, images are interpreted by the same reading centers, and laboratory specimens are processed at the same facilities. We scanned consecutive ESCAPE RA enrollees without contraindications to cardiac MRI until the predetermined subsample allotment was met. As we reported, there were no differences in clinical characteristics between RA patients who did and those who did not undergo cardiac MRI scanning.

Regarding potential incompatibility between the RA and control groups as a threat to the validity of our findings, we acknowledge that selection bias is a possibility in any study of this type. However, it is unlikely to be of sufficient magnitude to invalidate our findings, for several reasons. First, the main variables under study (cardiac MRI parameters) are subclinical, which decreases the likelihood that cases with abnormal values were included or selected. Additionally, through fre- 